\title{
Immunsuppression durch UV-Licht: rasch einsetzend und kräftig
}

Natürliche Sonnenstrahlung wird seit Jahrzehnten in der Therapie der Psoriasis genutzt. Ihre klinischen Wirkungen führt man neben allgemein günstigen Effekten einer kurmäßig angewandten Klimatherapie wie Stressabbau zumindest teilweise auf immunregulatorische Mechanismen zurück.

W as im Rahmen einer Sonnenlicht-Applikation auf zellulärer und molekularer Ebene passiert, wollten norwegische Immunologen und Rheumatologen genauer wissen. Daher untersuchten sie frühe sonneninduzierte immunologische Veränderungen bei Psoriasis-Patienten.

20 Patienten mit moderater bis schwerer Psoriasis ließen auf Gran Canaria für 15 Tage Sonne auf ihre Haut wirken. Vier Wochen zuvor mussten sie alle Antipsoriatika absetzen. Die kontrollierten Sonnenbäder induzierten eine signifikante klinische Verbesserung.

Für immunhistochemische Tests und die Bestimmung der Genexpression von Zytokinen entnahm man vor und während der Klimatherapie bei zehn Patienten Stanzbiopsien aus läsionaler Haut und aus nicht befallenen Hautarealen. Außerdem wurden Blutproben aller Teilnehmer auf T-Zell-Subpopulationen und Zytokin-Produktion untersucht.

Im Laufe der 15 Tage mit gezielter Sonnenexposition sank die Zahl der CD4- und CD8-T-Zellen in den Psoriasis-Herden signifikant, sowohl epidermal wie dermal.

Zumindest zum Teil könnte die beobachtete Abnahme der pathogenetisch wichtigen Zellen dadurch erklärt werden, dass sich im peripheren Blut bereits nach einem Tag Sonnenexposition signifikant weniger CLA-positive T-Zellen fanden. Das Oberflächenmolekül CLA (kutanes Lymphozyten-assoziiertes Antigen) ist für das „Homing“, also die Einwanderung zirkulierender T-Lymphozyten in die Haut verantwortlich.

Die Reduktion der T-Zellen wurde begleitet von einer geringeren Ausschüttung proinflammatorischer Zytokine. Auch in Kultur synthetisierten periphere mononukleäre Zellen, die den Patienten nach der Behandlung mit Sonnenlicht entnommen wurden, weniger Zytokine. Dieser Befund demonstriert, dass Sonnenlicht nicht nur lokale antientzündliche Effekte entfaltet, sondern auch die systemische Immunität moduliert.

Fazit: Die immunologischen Wirkungen des Sonnenlichts waren sehr rasch messbar. Da sie den gefundenen - exzellenten - Therapieerfolgen zeitlich vorausgingen, ist anzunehmen, dass die nachgewiesene Unterdrückung der lokalen und systemischen Entzündung für die klinischen Effekte verantwortlich ist. 